\title{
Anatomic and Angiographic Analyses of Ophthalmic Artery Collaterals in Moyamoya Disease
}

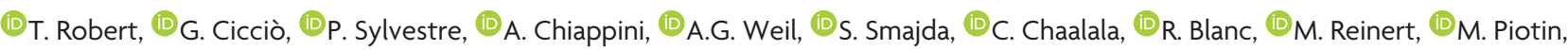
and (DM.W. Bojanowski

\begin{abstract}
BACKGROUND AND PURPOSE: Moyamoya disease is a progressive neurovascular pathology defined by steno-occlusive disease of the distal internal carotid artery and associated with the development of compensatory vascular collaterals. The etiology and exact anatomy of vascular collaterals have not been extensively studied. The aim of this study was to describe the anatomy of collaterals developed between the ophthalmic artery and the anterior cerebral artery in a Moyamoya population.
\end{abstract}

MATERIALS AND METHODS: All patients treated for Moyamoya disease from 2004 to 2016 in 4 neurosurgical centers with available cerebral digital subtraction angiography were included. Sixty-three cases were evaluated, and only 38 met the inclusion criteria. Two patients had a unilateral cervical internal carotid occlusion that limited analysis of ophthalmic artery collaterals to one hemisphere. This study is consequently based on the analysis of 74 cerebral hemispheres.

RESULTS: Thirty-eight patients fulfilled the inclusion criteria. The most frequently encountered anastomosis between the ophthalmic artery and cerebral artery was a branch of the anterior ethmoidal artery (31.1\%, 23 hemispheres). In case of proximal stenosis of the anterior cerebral artery, a collateral from the posterior ethmoidal artery could be visualized (16 hemispheres, $21.6 \%$ ). One case (1.4\%) of anastomosis between the lacrimal artery and the middle meningeal artery that permitted the vascularization of a middle cerebral artery territory was also noted.

CONCLUSIONS: Collaterals from the ophthalmic artery are frequent in Moyamoya disease. Their development depends on the perfusion needs of the anterior cerebral artery territories. Three other systems of compensation could be present (callosal circle, leptomeningeal anastomosis, and duro-pial anastomoses).

ABBREVIATIONS: $\mathrm{ACA}=$ anterior cerebral artery; OA = ophthalmic artery; STA = superficial temporal artery

M oyamoya disease is a neurovascular pathology characterized by the progressive stenosis of the supraclinoidal portion of the internal carotid artery and its 2 main branches: the anterior cerebral artery (ACA) and middle cerebral artery in their proximal segments. ${ }^{1-3}$ During the evolution of the pathology, the development of collaterals maintains a minimal perfusion to the cerebral parenchyma and, in particular, the ACA territories. ${ }^{3-5}$ In most cases, the ICA stenosis is distal to the ophthalmic artery (OA), ${ }^{1}$ thus allowing this artery to develop the necessary collater-

Received September 27, 2017; accepted after revision February 7, 2018.

From the Department of Interventional Neuroradiology (T.R., G.C., S.S., R.B., M.P.), Rothschild Foundation Hospital, Paris, France; University of Montreal (P.S.), Montreal, Quebec, Canada; Department of Neurosurgery (A.G.W., C.C., M.W.B.), NotreDame Hospital, Montreal, Quebec, Canada; and Department of Neurosurgery (T.R., A.C., M.R.), Neurocenter of Southern Switzerland, Lugano, Switzerland.

Please address correspondence to Thomas Robert, MD, Department of Neurosurgery, Neurocenter of the Southern Switzerland, Ospedale Civico di Lugano, Via Tesserete, 46, 6901 Lugano, Switzerland; e-mail: thomas.robert43@gmail.com

http://dx.doi.org/10.3174/ajnr.A5622 als. ${ }^{3}$ A detailed analysis of the role of the OA in supplying the ACA and MCA territories is lacking in the literature. By analyzing angiographic images, we aimed to study how the OA participates in creating collaterals in Moyamoya disease.

\section{MATERIALS AND METHODS Patient Selection}

On the basis of a prospective data base from 2004 to 2016 with demographics, 63 patients with the diagnosis of Moyamoya disease or syndrome have been evaluated in our institutions (Sainte Justine Hospital, Montreal; Notre-Dame Hospital, Montreal; Rothschild Foundation Hospital, Paris; and Regional Hospital, Lugano). In this study, the first digital subtraction angiography of these patients was available in only 38 cases. In 2 of these cases, unilateral cervical ICA stenosis limited our analysis of OA collaterals to only 1 hemisphere. Therefore, a retrospective analysis of OA collaterals was possible in 74 cerebral hemispheres. For each patient, additional data were collected retrospectively. We looked for the presence of an etiology, the duration of the symptoms, the 
Table 1: Demographic data and clinical presentation

\begin{tabular}{lc}
\multicolumn{1}{c}{ Variable } & $\begin{array}{c}\text { Patients } \\
(\boldsymbol{n}=38)\end{array}$ \\
\hline Age (median) (range) (yr) & $39.8(1-67)$ \\
Female & $28(73.7 \%)$ \\
Clinical presentation & \\
Ischemic stroke & $23(60.5 \%)$ \\
Hemorrhagic stroke & $9(23.7 \%)$ \\
Hemorrhagic stroke with intraventricular & $2(5.3 \%)$ \\
$\quad$ hemorrhage & \\
SAH secondary to aneurysm rupture & $3(7.9 \%)$ \\
Incidental discovery & $1(2.6 \%)$ \\
mRS at diagnosis & \\
1 & $12(31.6 \%)$ \\
2 & $15(39.5 \%)$ \\
3 & $4(10.5 \%)$ \\
4 & $1(2.6 \%)$ \\
5 & $6(15.8 \%)$ \\
\hline
\end{tabular}

type of clinical sign (ischemic or hemorrhagic), and the evaluation by a modified Rankin Scale score.

\section{Analysis of the Ophthalmic Artery Collateral Anatomy}

A 6-vessel DSA with the patient under local anesthesia with 3D reconstruction was conducted on all patients of this study. The Suzuki grading system ${ }^{6}$ was applied to each cerebral hemisphere; as suggested by Baltsavias et al, ${ }^{7}$ different arterial collaterals were categorized into leptomeningeal, duro-pial, and periventricular collaterals. For each ophthalmic artery evaluated, careful attention was paid to the presence of a rete mirabile modification of the $\mathrm{OA}$, to the presence of 1 or multiple anastomotic vascular networks between branches of the OA and branches of a cerebral artery, and to the cerebral territory supplied by branches of the OA.

\section{Treatment and Clinical Evolution}

Modalities of medical and surgical treatments were recorded. Follow-up started at the time of the first clinical sign and ended with the last visit. A neurologic examination with evaluation of the mRS was systematically performed for each visit.

\section{RESULTS}

\section{Clinical Presentation}

Between 2004 and 2016, thirty-eight patients fulfilled the inclusion criteria for this study. Patient baseline data and clinical signs are described in Table 1 . The mean age was 40 years (range, 1-67 years) with a female preponderance (female/male ratio: 2.8:1). The most common clinical sign was an ischemic stroke in 23 patients $(60.5 \%)$. Other patients presented with bleeding: intraparenchymal hematoma ( 9 patients, $23.7 \%$ ), intraparenchymal bleeding with a ventricular component (2 patients, 5.3\%), and a subarachnoid hemorrhage secondary to an associated aneurysm rupture in 3 patients (7.9\%). In 1 patient, Moyamoya disease was discovered incidentally while investigating a frontal parasagittal meningioma. The initial mRS score was 1 in 12 patients $(31.6 \%)$, 2 in $15(39.5 \%), 3$ in $4(10.5 \%), 4$ in 1 (2.6\%), and 5 in $6(15.8 \%)$.

\section{Angiographic Analysis of Moyamoya Disease}

We analyzed the ophthalmic arteries and their collaterals to the cerebral parenchyma for each cerebral hemisphere. It was not
Table 2: Angiographic Suzuki grade and presence of collaterals

\begin{tabular}{lc}
\hline \multicolumn{1}{c}{ Cerebral Hemispheres Studied } & $\boldsymbol{n}=74$ \\
\hline Suzuki stage & $9(12.2 \%)$ \\
I & $10(13.5 \%)$ \\
II & $10(13.5 \%)$ \\
III & $14(18.9 \%)$ \\
IV & $15(20.3 \%)$ \\
V & $5(6.8 \%)$ \\
VI & $11(14.9 \%)$ \\
OA-intracranial artery collaterals & $35(47.3 \%)$ \\
Rete mirabile of the OA & $17(23 \%)$ \\
Anterior ethmoidal artery-ACA branch direct & $15(20.3 \%)$ \\
$\quad$ collaterals & $23(31.1 \%)$ \\
Anterior ethmoidal artery-ACA branch collaterals & \\
$\quad$ via anterior falcine artery & $16(21.6 \%)$ \\
Posterior ethmoidal artery-orbitofrontal artery & $1(1.4 \%)$ \\
Recurrent meningeal artery-MMA-Sylvian branch & \\
Collaterals & \\
$\quad$ Leptomeningeal collaterals to ACA territory & $51(68.9 \%)$ \\
Duro-pial collaterals to ACA territory & $5(6.8 \%)$ \\
Posterior callosal artery collateral & $22(29.7 \%)$ \\
Periventricular collaterals & $47(63.5 \%)$ \\
\hline
\end{tabular}

Note:-MMA indicates middle meningeal artery.

possible to assess the $\mathrm{OA}$ in 2 patients due to a unilateral cervical ICA occlusion. The results of our evaluation are summarized in Table 2. The Suzuki stage was 0 in 9 patients presenting with a unilateral Moyamoya syndrome (12.2\%), I in 10 hemispheres (13.5\%), II in $10(13.5 \%)$, III in 14 sides (18.9\%), IV in 15 (20.3\%), V in $5(6.8 \%)$, and VI in 11 hemispheres (14.9\%). Leptomeningeal collaterals were the most frequently observed (51 hemispheres, 68.9\%). Duro-pial collaterals from branches of the external carotid artery to the territory of the ACA were noted in 5 hemispheres $(6.8 \%)$, and periventricular collaterals, in 47 sides (63.5\%). The presence of collaterals from branches of the ophthalmic artery was noted in 35 cases $(47.3 \%)$ and was most frequent in Suzuki grades III-V. A rete mirabile modification of the ophthalmic artery was present in 17 cases (23\%).

\section{Collaterals from the Anterior Ethmoidal Artery}

The most frequently encountered anastomosis between the ophthalmic artery and a cerebral artery was a branch of the anterior ethmoidal artery. It was found in $31.1 \%$ (23 hemispheres). Figure 1 illustrates one of these cases. The anterior ethmoidal artery supplies the anterior falcine artery, which develops a duro-pial collateral with the callosomarginal, anteromedial frontal, or frontopolar artery. These collaterals could supply the territory of the anterior cerebral artery when there was poor development of collaterals from the posterior callosal artery.

\section{Collaterals from the Posterior Ethmoidal Artery}

In 16 hemispheres $(21.6 \%)$ in our series, when there was a proximal stenosis of the anterior cerebral artery, a collateral from the posterior ethmoidal artery was seen. The posterior ethmoidal artery supplied the paramedian part of the planum sphenoidale, and by its proximity, it developed a collateral with the homolateral orbitofrontal artery. By retrograde flow, the orbitofrontal artery supplied the A2 segment of the ACA. Figure 2 shows an example of this collateral artery. 


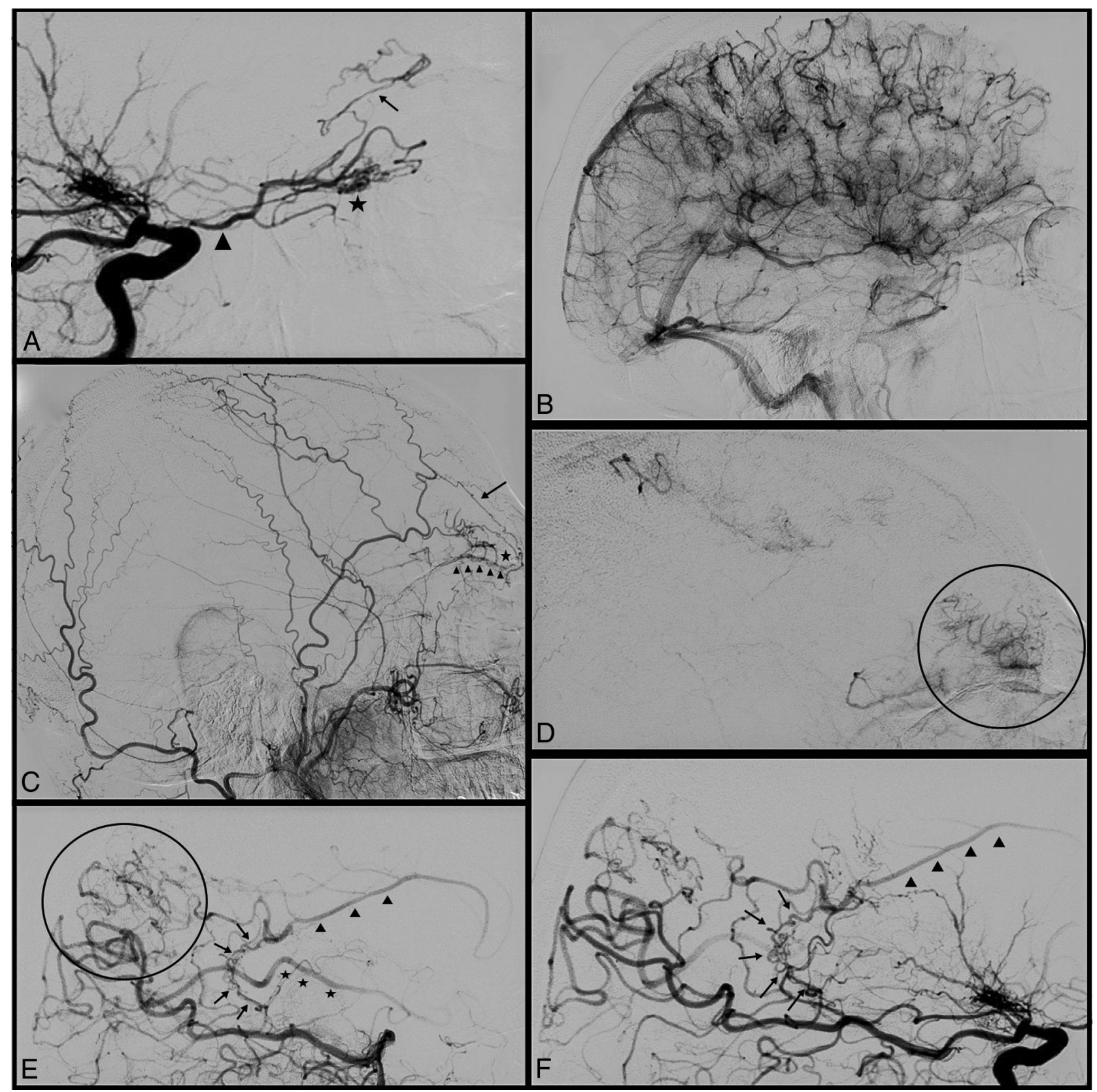

FIG 1. Illustrative case 1. A, Lateral ICA angiogram with the ophthalmic artery (triangle) with a rete mirabile modification of its third segment (asterisk) and an anterior ethmoidal artery anastomosis with retrograde perfusion of an anterior cerebral artery branch (black arrow). $B$, Parenchymal time of the angiogram in $A$ shows the complete perfusion of the frontal lobe. C, Lateral external carotid artery angiogram of the same patient shows the presence of an anastomosis between the ophthalmic artery and the superficial temporal artery (black arrow) via the dorsal nasal artery. Note the presence of anterior ethmoidal artery anastomosis with pial branches of the anterior cerebral artery (asterisk). The arrowheads show anastomotic branches under the orbital roof. $D$, Parenchymal time of the angiogram in $C$ that shows the perfusion of the frontopolar region by the anastomosis described earlier. E, An example of retrograde perfusion of the pericallosal artery (arrowheads) on the lateral vertebral artery angiogram by callosal circle (arrows) and cortical leptomeningeal anastomoses. Note also the presence of retrograde opacification of an MCA branch (asterisks). F, Another example of retrograde perfusion of the pericallosal artery (arrowheads) on a lateral vertebral artery angiogram by a well-developed callosal circle (arrows).

\section{Collaterals from the Lacrimal Artery}

In only 1 case in our series (1.4\%) did the ophthalmic artery supply a part of the MCA territory through the recurrent meningeal artery. The anterior branch of the middle meningeal artery originated from the lacrimal artery and passed through the superior orbital fissure. This branch of the middle meningeal artery developed duro-pial collaterals with cortical branches of the MCA.

\section{Treatment and Clinical Follow-Up}

Among the 38 patients, 21 were treated with an antiplatelet therapy. Aspirin was always the first-line treatment. Three of them presented with a recurrent ischemic stroke and were placed under dual-antiplatelet therapy. No hemorrhagic stroke was noted under antiaggregation therapy. Six patients benefited from surgical treatment. Six patients had a bilateral superficial temporal artery (STA)-middle cerebral artery anastomosis, and 4 others had a 


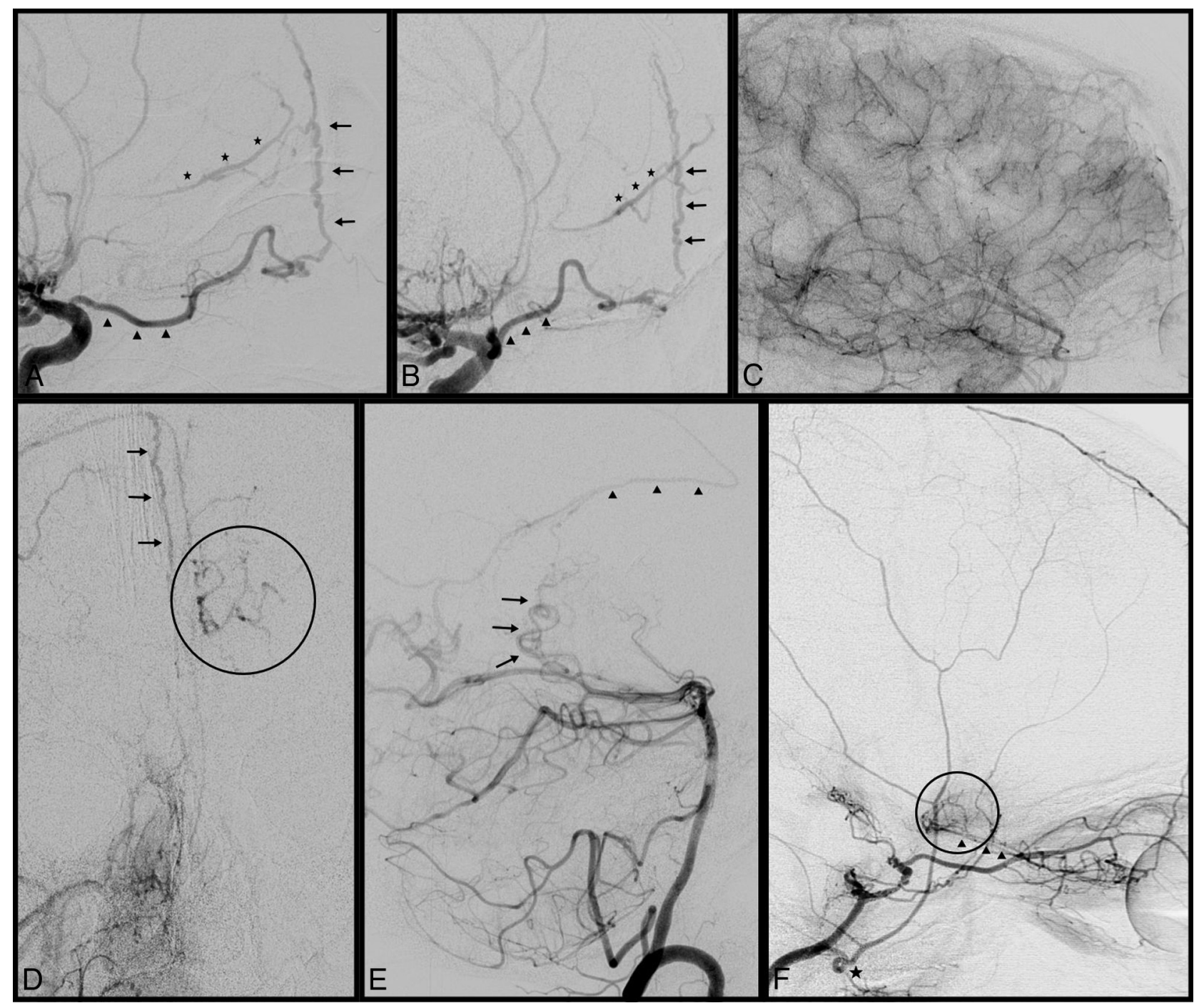

FIG 2. Illustrative case 2. A, Lateral ICA angiogram with the ophthalmic artery (triangles) having an anterior ethmoidal artery anastomosis by a falcine artery (arrows) with retrograde perfusion of an anterior cerebral artery branch (asterisks). The same angiogram as shown in $A$ in an oblique view. $C$, Parenchymal time of the angiogram of $A$ and $B$ that shows the complete perfusion of the frontal lobe. $D$, An example of a duro-pial anastomosis from the right middle meningeal artery via the falcine arteries (arrows) to the left cingulate gyrus. $E$, The presence of retrograde perfusion of the pericallosal artery (arrowheads) on a lateral vertebral artery angiogram by a callosal circle (arrows). F, Lateral ICA angiogram with complete occlusion of the supraclinoidal segment of the ICA and posterior ethmoidal artery anastomosis (arrowheads). Note the presence of an ICA origin of the middle meningeal artery (asterisk).

STA-MCA anastomosis only at 1 side. For the last patient, the STA was too small to permit the anastomosis and a myosynangiosis was performed. As a complication of the operation, 1 patient had an ischemic stroke contralateral to the operated hemisphere that was imputed to a hypoperfusion during the induction. The mean follow-up was 50 months (range, 1-138 months). At the last visit, 8 patients $(40 \%)$ had a poor clinical outcome $(\mathrm{mRS} \geq 3)$ that was directly correlated to the clinical presentation of the patient before treatment.

\section{DISCUSSION}

Moyamoya disease is a progressive neurovascular pathology defined by stenosis of the distal internal carotid artery and middle and anterior cerebral arteries associated with the development of vascular collaterals. The scientific literature regarding this pathology is limited because of the rarity of Moyamoya disease. ${ }^{4}$ Most articles described the surgical techniques and their outcome, but only a few authors provided detailed angiographic descriptions of the different types of collaterals that naturally develop. In particular, Baltsavias et $\mathrm{al}^{7}$ described the collaterals that had developed in the anterior and posterior ${ }^{8}$ circulations. They classified the different anastomosis into 4 types using superselective angiography. Although it is known that the OA may provide collaterals, a detailed analysis is not available in the literature, to our knowledge. A lot of questions remain concerning these collaterals: Are they frequent? Is there a pattern? Do certain collaterals develop more frequently than others? What are the other possibilities of maintaining blood flow to territories of the anterior cerebral arteries?

The most frequent collaterals found in our series came from branches of the anterior ethmoidal artery. Normally, this artery vascularizes the dura of the medial third of the anterior fossa and the anterior third of the falx cerebri, the latter via the anterior 

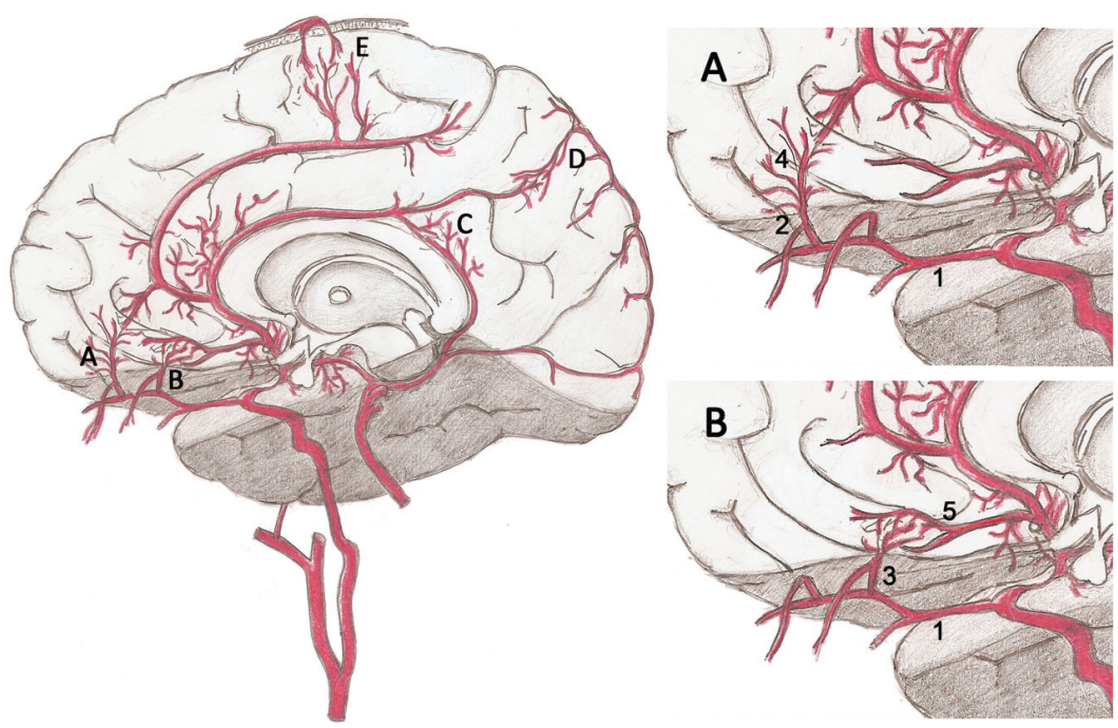

FIG 3. Illustration showing the 4 systems of arterial collaterals to supply the ACA territories. $A$, Anterior ethmoidal artery anastomosis (1: ophthalmic artery; 2: anterior ethmoidal artery; 4: anterior falcine artery). B, Posterior ethmoidal artery anastomosis (1: ophthalmic artery; 3 : posterior ethmoidal artery; 5: orbitofrontal artery). C, Callosal circle. D, Leptomeningeal anastomosis (posterior cerebral artery-ACA). E, Duro-pial anastomosis from a branch of the middle meningeal artery.

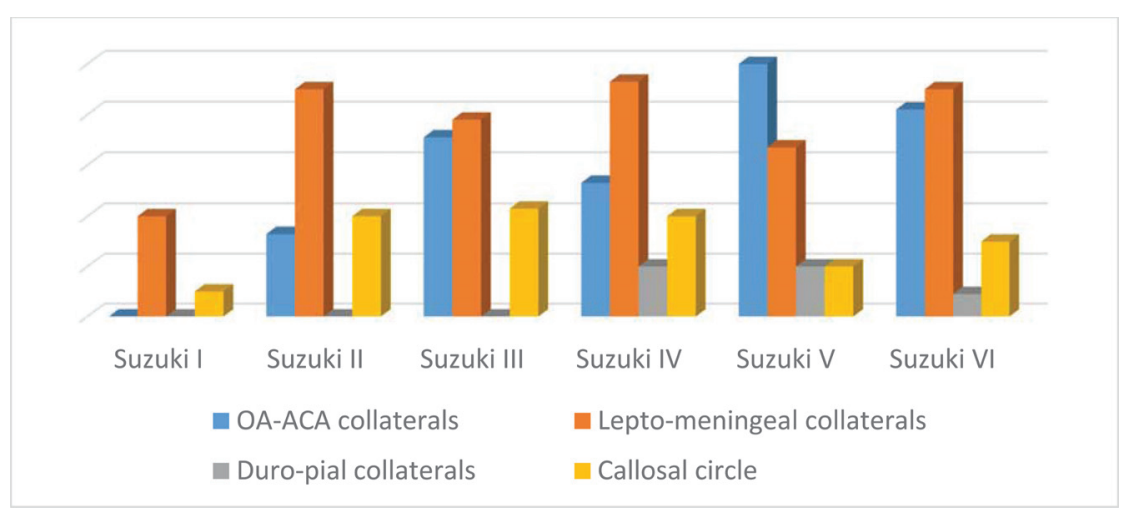

FIG 4. Evolution of the systems that permit the perfusion of the ACA territories as a function of the Suzuki grade.

falcine artery. Baltsavias et $\mathrm{al}^{7}$ described duro-pial anastomoses between the middle meningeal artery and cortical branches. In their description of the duro-pial anastomoses, they gave an example of an anastomosis between an anterior ethmoidal artery and an orbitofrontal artery. ${ }^{7}$ These small collaterals help in the perfusion of a callosomarginal artery from a well-developed anterior falcine artery.

Another type of collateral described in our series is a connection between the posterior ethmoidal artery and a proximal branch of the anterior cerebral artery. This less frequent anastomosis has a posteriorly oriented trajectory and may perfuse retrogradely an artery that originates near the anterior communicating complex. This anastomosis has already been described by Chung and Weon ${ }^{9}$ in a case report in which the authors described the rete mirabile appearance of the ophthalmic artery. We have 2 hypotheses to explain the development of this anastomosis. First, we suggest that this anastomosis is a duro-pial anastomosis between a dural branch of the posterior ethmoidal artery and the orbitofrontal artery. This orbitofrontal artery is in the olfactory sulcus and takes its origin from the $\mathrm{A} 2$ near the A1-A2 junction. A retrograde flow from the posterior ethmoidal artery may supply the entire pericallosal artery. The second hypothesis takes into account the presence of the remnant of the primitive olfactory artery, an embryologic artery described by Padget. ${ }^{10}$ She described the primitive olfactory artery as the anterior branch of the primitive internal carotid artery. Its maximal development is the sixth stage $(20-24 \mathrm{~mm})$ when it is an A1 branch that follows the olfactory tract to the posterior part of the cribriform plate. This artery then enters the ethmoidal cells and the orbit to anastomose with ophthalmic artery branches. After this stage, the medial part of the artery regresses, the distal part becomes the dural branches of the posterior ethmoidal branch, and the proximal part develops into a recurrent artery that enters the anterior perforated substance. After comparing their results with the works of Abbie ${ }^{11}$ and Shellshear, Padget ${ }^{10}$ considered the primitive olfactory artery as the embryologic precursor of the recurrent artery of Heubner. Lasjaunias et $\mathrm{al}^{12}$ cited the interesting works of Padget and added phylogenic information. In horses and monkeys, the anterior cerebral artery is supplied by the ophthalmic artery through an artery that courses into the posterior part of the cribriform plate.

The last type of collateral observed in our series is the supply of the territory of the middle cerebral artery by the ophthalmic artery via the middle meningeal artery. This anastomosis is only possible with the persistence or the development of the recurrent meningeal artery. The recurrent meningeal artery is an anastomotic artery between the lacrimal artery and the middle meningeal artery that is the remnant of the orbital branch of the stapedial artery. Uchino et $\mathrm{al}^{13}$ and Komiyama et $\mathrm{al}^{14}$ have observed that the incidence of an embryonic remnant of an artery is most frequent in patients with Moyamoya disease and suggested a congenital origin of the pathology. With only 1 case of this anastomosis noted in our series, we have not reached any conclusions, but it remains an interesting observation.

In the present series, only a few cases of Moyamoya disease in the first stages present with vascular collaterals from the ophthalmic artery. We also noted that in Suzuki stage III, most cases have already developed OA-ACA collaterals. These collaterals seem to be functional earlier than the duro-pial collaterals developed from the middle meningeal artery. In advanced stages of the disease (Suzuki V and VI), the proportion of OA-ACA collaterals is high.

It is very difficult to understand which factors influence the 
development of OA-ACA collaterals. Our impression is that there is a lack of blood supply, which stimulates the development of collaterals. We identified no more than 4 systems that allow maintaining the supply of the ACA territories. These 4 systems are illustrated in Fig 3. The first one is the callosal circle (Fig 3A), which is the anastomosis of the posterior and anterior callosal arteries. The second one is the leptomeningeal collaterals (Fig 3B) from cortical arteries of the MCA or posterior cerebral artery. The third one is duro-pial anastomosis (Fig 3C) from the middle meningeal artery, and the last one, OA-ACA anastomosis (Fig 3D). A balance among these 4 systems permitting maintenance of the cerebral perfusion seems to be the most important factor explaining the development of OA-ACA collaterals. The balance among these 4 systems found in our series is shown in Fig 4. Baltsavias et $\mathrm{al}^{7}$ described another possible system that could supply the ACAs, the development of transcallosal anastomosis from the choroidal to the pericallosal arteries.

Few articles ${ }^{2-4,7,8,12}$ elucidate the precise anatomy of collaterals developed in the Moyamoya disease. The sequence of the development is not known, and the only information we have is summarized in the Suzuki score. ${ }^{6}$ We think that the understanding of the evolution of these collaterals is key to managing these patients. With enough information, it could be easier to select patients who need bypass surgery and determine the best time to perform it.

\section{Limitations}

Our study is a retrospective study based on conventional cerebral DSA without selective injection to evaluate the anastomosis between the OA and the cerebral arteries. The other important limitation is that most of our patients had only 1 conventional DSA and thereafter were followed by MRA. It is impossible to evaluate the development of these collaterals during the evolution of the Moyamoya disease.

\section{CONCLUSIONS}

Collaterals from the ophthalmic artery are frequent in Moyamoya disease, particularly at Suzuki stage III. Their development depends on the lack of blood supply to the ACA territories as well as on the ability of the 3 other systems (callosal circle, leptomenin- geal anastomosis, and duro-pial anastomoses) to compensate. Selective injection of the $\mathrm{OA}$ at various stages of the disease may help to better understand the dynamics involved in the development of collaterals from the OA in Moyamoya disease.

\section{REFERENCES}

1. Scott RM, Smith ER. Moyamoya disease and moyamoya syndrome. N Engl J Med 2009;360:1226-37 CrossRef Medline

2. Bang OY, Fujimura M, Kim SK. The pathophysiology of Moyamoya disease: an update. J Stroke 2016;18:12-20 CrossRef Medline

3. Burke GM, Burke AM, Sherma AK, et al. Moyamoya disease: a summary. Neurosurg Focus 2009;26:E11 CrossRef Medline

4. Kim JS. Moyamoya disease: epidemiology, clinical features, and diagnosis. J Stroke 2016;18:2-11 CrossRef Medline

5. Kwag HJ, Jeong DW, Lee SH, et al. Intracranial hemodynamic changes during adult moyamoya disease progression. J Clin Neurol 2008;4:67-74 CrossRef Medline

6. Suzuki J, Takaku A. Cerebrovascular "moyamoya" disease: disease showing abnormal net-like vessels in base of brain. Arch Neurol 1969;20:288-99 CrossRef Medline

7. Baltsavias G, Khan N, Valavanis A. The collateral circulation in pediatric moyamoya disease. Childs Nerv Syst 2015;31:389-98 CrossRef Medline

8. Baltsavias G, Khan N, Filipce V, et al. Selective and superselective angiography of pediatric moyamoya disease angioarchitecture in the posterior circulation. Interv Neuroradiol 2014;20:403-12 CrossRef Medline

9. Chung JI, Weon YC. Ophthalmic rete mirabile: the first angiographic documentation of embryonic ophthalmic collaterals in a patient with moyamoya disease: a case report. Interv Neuroradiol 2008;14:293-96 CrossRef Medline

10. Padget DH. The development of the cranial arteries in the human embryo. Contrib Embryol 1948;32:205-62

11. Abbie AA. The morphology of the fore-brain arteries, with especial reference to the evolution of the basal ganglia. J Anat 1934;68: 433-70 Medline

12. Lasjaunias P, Brugge KG, Berenstein A. Surgical Neuroangiography. New York: Springer-Verlag; 2006

13. Uchino A, Saito N, Takahashi M, et al. Cerebral arterial variations associated with Moyamoya disease diagnosed by MR angiography. Neuroradiol J 2014;27:697-701 CrossRef Medline

14. Komiyama M, Nakajima $H$, Nishikawa $M$, et al. High incidence of persistent primitive arteries in moyamoya and quasi-moyamoya diseases. Neurol Med Chir (Tokyo) 1999;39:416-20; discussion 420-22 CrossRef Medline 